\title{
Hybrid random walk-linear discriminant analysis method for unwrapping quantitative phase microscopy images of biological samples
}

Diane N. H. Kim

Michael A. Teitell

Jason Reed

Thomas A. Zangle 


\title{
Hybrid random walk-linear discriminant analysis method for unwrapping quantitative phase microscopy images of biological samples
}

\author{
Diane N. H. Kim, ${ }^{a}$ Michael A. Teitell, ${ }^{\text {a,b,c,d,e,f Jason Reed, }{ }^{g, h} \text { and Thomas A. Zangle }}{ }^{\text {a,b,c,* }}$ \\ aUniversity of California, Los Angeles, Department of Bioengineering, TLSB 3126, California 90095, United States \\ bUniversity of California, Los Angeles, Jonsson Comprehensive Cancer Center, 8-950 Factor Building, California 90095, United States \\ 'University of California, Los Angeles, Broad Stem Cell Research Center, Box 957357, California 90095, United States \\ dUniversity of California, Los Angeles, David Geffen School of Medicine, Department of Pathology and Laboratory Medicine, MRL 4762, California \\ 90095, United States \\ eUniversity of California, Los Angeles, NanoSystems Institute, 570 Westwood Plaza, California 90095, United States \\ fUniversity of California, Los Angeles, Molecular Biology Institute, Box 951570, California 90095, United States \\ gVirginia Commonwealth University, Department of Physics, 701 West Grace Street, Richmond, Virginia 23284, United States \\ hVirginia Commonwealth University, Massey Cancer Center, 401 College Street, Richmond, Virginia 23284, United States
}

\begin{abstract}
Standard algorithms for phase unwrapping often fail for interferometric quantitative phase imaging (QPI) of biological samples due to the variable morphology of these samples and the requirement to image at low light intensities to avoid phototoxicity. We describe a new algorithm combining random walk-based image segmentation with linear discriminant analysis (LDA)-based feature detection, using assumptions about the morphology of biological samples to account for phase ambiguities when standard methods have failed. We present three versions of our method: first, a method for LDA image segmentation based on a manually compiled training dataset; second, a method using a random walker (RW) algorithm informed by the assumed properties of a biological phase image; and third, an algorithm which combines LDA-based edge detection with an efficient RW algorithm. We show that the combination of LDA plus the RW algorithm gives the best overall performance with little speed penalty compared to LDA alone, and that this algorithm can be further optimized using a genetic algorithm to yield superior performance for phase unwrapping of QPI data from biological samples. $\odot$ The Authors. Published by SPIE under a Creative Commons Attribution 3.0 Unported License. Distribution or reproduction of this work in whole or in part requires full attribution of the original publication, including its DOI. [DOI: 10.1117/1.JBO.20.11.111211]
\end{abstract}

Keywords: quantitative phase imaging; quantitative phase microscopy; phase unwrapping; image processing; cell biology. Paper 150148SSR received Mar. 12, 2015; accepted for publication Jul. 22, 2015; published online Aug. 25, 2015.

\section{Introduction}

There is increasing interest in the application of quantitative phase imaging (QPI) as a high-sensitivity method to study the structural and dynamic behavior of biological samples. ${ }^{1,2}$ These samples typically consist of single cells or cell clusters, which are imaged over time to track changes in dry mass ${ }^{3}$ or refractive index, ${ }^{4,5}$ for example, to monitor cellular growth ${ }^{6-8}$ and growth regulation, ${ }^{9}$ quantify intercellular interactions ${ }^{10}$ or subcellular features,${ }^{11}$ and measure mechanical properties of cells, such as red blood cells. ${ }^{12-14}$ In any interferometric QPI dataset, the phase ambiguities inherent to QPI have to be removed to achieve continuous phase distributions and precise, reproducible measurements of these samples. ${ }^{15}$

Basic phase unwrapping of large jump discontinuities are easily handled by a variety of algorithms, including the Flynn or widely used Goldstein method. ${ }^{15}$ These algorithms can be classified into three broad groups: path-following algorithms, region algorithms, and global algorithms. ${ }^{16}$ In path-following algorithms, phase integration is performed in a step sequence. Goldstein's branch cut algorithm is an example of a pathdependent path-following algorithm that identifies inconsistency-

*Address all correspondence to: Thomas A. Zangle, E-mail: tzangle@ucla.edu causing structures called residues and connects and balances them. ${ }^{17}$ Region algorithms divide the data field into separate regions, which are individually processed. The regions are then unwrapped with respect to each other to process the entire image. ${ }^{18}$ Flynn's mask cut algorithm is an example of a region algorithm. ${ }^{15,19}$ Global algorithms formulate phase unwrapping in terms of minimization of a global function..$^{15,20}$ More recent alternative algorithms have built on or modified these classic methods. $^{18,21}$

However, imaging biological samples presents a unique challenge because of the varying morphology of cells during cell culture, combined with the fact that the optical path length through cells relative to background levels routinely exceeds $2 \pi$ rad. Due to the varying morphology of cells and the desire for high throughput measurements, these large phase differences can occasionally occur over distances spanning a single pixel in the resulting image. This is especially problematic for nonadherent mammalian cells or for adherent mammalian cells during mitosis, which typically have a large jump in phase from the edge of the cell to the surrounding background. These edges are liable to be phase unwrapped incorrectly by standard algorithms [Fig. 1(a)]. Additionally, to avoid phototoxicity when imaging live cells, ${ }^{22}$ a low light intensity is preferable, which results in poor phase quality and high relative noise levels, ${ }^{23}$ further complicating phase unwrapping. 

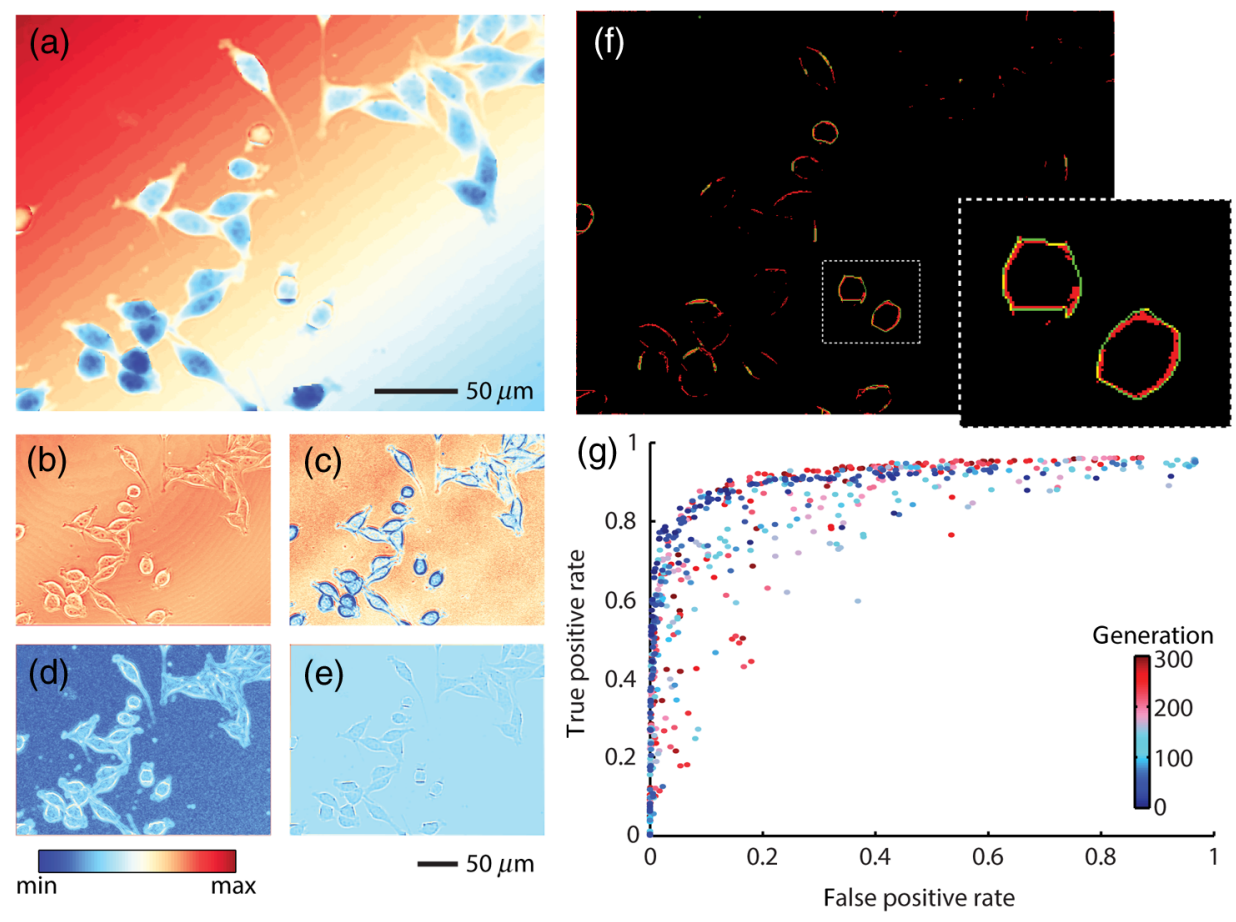

Fig. 1 Linear discriminant analysis (LDA)-based segmentation for phase-unwrapping of biological sample quantitative phase imaging data. (a-e) selected inputs to LDA method (a) raw (unwrapped) phase data, (b) intensity image, (c) phase modulation magnitude, (d) natural logarithm of the phase derivative variance, (e) Laplace-filtered phase image, and (f) predicted edges from LDA composite image compared to manual phase unwrapping results. In (f), green shows the manually selected mask edge, red is the LDA detected edge, and yellow shows overlap of both edges. $(\mathrm{g})$ True positive rate versus false positive rate for LDA-based image segmentation during successive optimizations by a genetic algorithm (GA). The ratio of false positives to true positives generally decreases with increased number of generations with some variation inherent to genetic optimization algorithms.

There are a large variety of methods that have been proposed to address this problem for two-dimensional images of biological samples. Some methods bypass the unwrapping process itself through the modification of instrumentation, including heterodyne mixing, ${ }^{24}$ optical path locking, ${ }^{25}$ and the use of the transport of intensity equation. ${ }^{26,27}$ However, for many of the available QPI methods, phase unwrapping remains a general problem of interest. There are several alternative algorithms that modify the classic phase unwrapping methods or use specially developed software to improve the speed and throughput of phase unwrapping for QPI of biological specimens. ${ }^{18,28}$ However, these methods are largely based on the standard algorithms and may not adequately address all phase unwrapping problems faced by these methods.

Here, we present a new phase unwrapping method for correcting phase ambiguities in QPI of biological samples where the standard algorithms have failed. This approach, a hybrid random walk-linear discriminant (HRL) method, is intended to be applied to phase-shifting interferometry (PSI) data after first applying the Goldstein algorithm. ${ }^{15,17}$ The HRL approach combines two techniques: linear discriminant analysis (LDA), a widely used scheme for dimension reduction and feature extraction, and a random walker (RW) segmentation algorithm. LDA projects data onto a lower dimensional vector space such that the ratio of between-class difference to the within-class difference is maximized, achieving maximum discrimination between classes. ${ }^{29}$ In this application, it is used to emphasize the edges of phase-wrapped regions. In RW image segmentation, a seed mask specifies locations in the image with a predetermined label. Unseeded pixels are labeled using the probabilities that a RW agent starting at that location terminates at each label in the seed mask. The random walk can be biased (for example, to avoid sharp intensity gradients), and the final segmentation is derived by determining the most probable seed destination for each pixel. ${ }^{30}$ Here, the LDA class probability image is used as the weighting function for the RW algorithm to efficiently segment phase-wrapped regions.

The HRL algorithm combines the advantages of these two techniques based on two assumptions about QPI data from biological samples: (1) cell optical path length is never less than zero $\left(n_{\text {cell }}>n_{\text {media }}\right)$ and (2) uncorrected regions are predominantly $2 \pi$ rad lower than the correct level, as is commonly observed in our QPI datasets of biological samples. This second assumption means that the phase unwrapping problem is reduced to a matter of classifying pixels as belonging to properly phase unwrapped segments ("good" regions) or improperly unwrapped segments ("bad" regions) and applying a one-wavelength correction to pixels in the "bad" regions. To achieve this, manually phase-unwrapped training data is used to train an LDA classifier to generate an image that highlights the edges of phase-wrapped regions. Then, a RW segmentation method is applied to this image, starting at known-good or known-bad points based on the assumption that the cell optical path length is never less than zero. This segmentation approach is highly efficient because the gradient of the LDA image is large at the boundaries of the phase-wrapped regions, and the RW method is, therefore, strongly biased to not cross these boundaries. As a final step, a genetic algorithm (GA) is applied to 
fine-tune the LDA coefficients. The result is phase unwrapping that is highly accurate relative to manually unwrapped results [area under curve $(\mathrm{AUC})=0.999$ ] for effective phase unwrapping of biological phase interferometry data.

\section{Methods}

\subsection{Quantitative Phase Imaging Data}

Phase, intensity, and phase modulation magnitude ${ }^{31}$ data were acquired on a Contour GT-X8 (Bruker) interference microscope in PSI mode. The Bruker system was modified to accommodate a custom live cell incubation system and a $20 \times, 0.28$ numerical aperture microscope objective with attached Michelson interferometer. The Michelson interferometer consisted of a beam splitter, reference mirror, and compensation reference chamber filled with deionized water. Illumination was provided by a $530-\mathrm{nm}$ fiber-coupled LED (Thorlabs). Mouse L-cell fibroblasts and M202 human melanoma cells ${ }^{32}$ were cultured as described previously ${ }^{7,10,33}$ in phenol-red free Dulbecco's modified Eagle's medium supplemented with $10 \%$ fetal bovine serum (Omega Scientific), $1 \times$ nonessential amino acid solution (Invitrogen), $2 \mathrm{mM}$ glutamine (Invitrogen) and antibiotics.

Raw interferograms were unwrapped using the Goldstein method, ${ }^{17}$ which unwraps images by isolating and avoiding local-error-causing residues, ${ }^{15}$ prior to the manual or automated correction methods described here.

\subsection{Manual Phase Unwrapping}

Manual phase unwrapping was performed on a set of 50 phase images of mouse L-cells and 50 phase images of M202 melanoma cells to serve as training datasets and 50 additional images of each cell type as test sets for evaluation of our final automatic phase unwrapping method. Regions to be corrected were selected by looking for cellular areas with sharp internal edges, indicating a poorly placed branch cut in the Goldsteinunwrapping process, or regions with phase shifts below the background level, which would indicate nonphysical refractive indices less than that of the cell culture media. A custom graphical user interface was written in MATLAB (Mathworks) with three main steps: (1) select an integer multiple of $2 \pi$ rad correction to be applied, (2) select image regions to be corrected using the imfreehand function in the image processing toolbox, and (3) manually correct the phase unwrapping result at individual points. This process was repeated until all phase-wrapped regions were corrected. Results were then saved as a mask file containing the integer-wavelength corrections, as well as a corrected phase map.

\subsection{Linear Discriminant Analysis}

LDA was performed pixelwise based on 17 parameters: (1) the raw phase data after Goldstein unwrapping, (2) the estimated image background constructed by masking the location of any apparent cells, then fitting a plane to the remaining background regions to remove any tilt, (3) a Prewitt filtered image, (4) a Sobel filtered image; (5) a Laplacian filtered image with kernel [0 - $0 ;-14-1 ; 0-10],(6)$ a modified Laplacian filtered image with kernel $[-0.5-1-0.5 ;-18-1 ;-0.5-1-0.5]$, (7) a large Laplacian filtered image with kernel $[-10-5-2$ $-1-2-5-10 ;-503430-5 ;-236763-2 ;-147$ $874-1 ;-2366763-2 ;-5034430-5 ;-10-5-2$ $-1-2-5-10]$, (8) a Laplacian of Gaussian filtered image, ${ }^{34}$
(9) a $3 \times 3$ mean filtered image, (10) a $3 \times 3$ median filtered image, (11) the phase derivative variance (PDV) of the image, defined as the variance of the partial derivative of the phase in a four-connected neighborhood at each pixel and computed using the method described in the phase quality guided path following method ${ }^{15}$ and implemented in MATLAB by Spottiswoode, ${ }^{35}$ (12) the natural logarithm of the PDV image to provide data with comparable dynamic range to the other measures, (13) the phase modulation magnitude, or amplitude of the intensity fluctuations in the phase-shifted fringes, computed by the Bruker optical profilometer, ${ }^{31}$ (14) the natural logarithm of the phase modulation image, (15) the Laplacian filtered phase modulation image, (16) the intensity image, and (17) the Laplacian filtered intensity image.

LDA class probabilities were calculated using the Fisher linear discriminant ${ }^{36}$ with prior probabilities equal to the observed sample probabilities and all code implemented in MATLAB. ${ }^{37}$ The LDA-only phase unwrapping algorithm used LDA class probabilities as the input to a marker-based watershed algorithm ${ }^{34}$ to define phase-wrapped image regions.

\subsection{Random Walker Algorithm}

The RW algorithms assign each pixel a probability that a RW agent released from that pixel will reach a predetermined pixel of a given label (e.g., known-good or known-bad). Pixel labels were assigned by first subtracting the background tilt from raw phase images. Then pixels were assigned a "bad" label if they were more than a threshold value below the background level of zero, or if they were on the low side of an edge with a first derivative greater than a jump threshold (computed in all four cardinal directions by first-order discretization). Pixels were assigned a "good" label if they were smaller than the mean phase value in their $100 \times 100$ pixel neighborhood or if they were on the high side of an edge with a first derivative greater than the jump threshold.

The faster random walk method was based on the algorithm presented and implemented in MATLAB by Grady. ${ }^{30}$ Briefly, this method bypasses a Monte Carlo-type simulation of individual RW agents by solving for a solution to the Dirichlet problem. ${ }^{30,38,39}$ Additionally, this method can be biased to obtain higher quality segmentation by adjusting the probability that a RW agent will move in a given direction based on the difference in image intensity between the two corresponding pixels. To bias this method for more accurate segmentation, we tested the phase image, the LDA class probability image, and the natural logarithm of the LDA class probability image, with the natural logarithm of the LDA class probability image giving superior results.

\subsection{Genetic Algorithm}

LDA images define the probability that a given pixel lies on the edge of a phase-wrapped region, but does not compute the optimal image statistic weighting required for follow-on processing to turn those probability images into correctly phase-unwrapped data. To optimize LDA coefficients for phase unwrapping by watershed transform or the RW algorithm, we searched for an optimal set of coefficients to the LDA terms using the GA from the global optimization toolbox in MATLAB. The GA is an iterative process that repeatedly modifies a population of individual solutions. Random individuals are selected at each step and used to produce the next iteration. Over successive 
generations, the optimal solution is reached [Fig. 1(g)]. We used a genetic optimization with a tolerance of $1 \times 10^{-10}$, a migration fraction of 0.3 and an initial range of -10 to 10 for all LDA term coefficients. For RW methods, we also modified the background threshold parameter with an initial range of 0.06 to $1.57 \mathrm{rad}$.

\section{Results}

\subsection{Linear Discriminant Analysis Segmentation}

We manually unwrapped a randomly chosen set of 50 images of mouse L-cells and a set of 50 images of M202 human melanoma cells, which contained phase unwrapping errors after phase unwrapping via the Goldstein method. In the mouse L-cell image set, $1.2 \%$ of pixels in this set were phase wrapped, and the vast majority of these errors $(99.7 \%)$ were $2 \pi$ rad lower than the expected phase value, with the remaining $0.3 \%$ of phase errors being $2 \pi$ rad too high. We chose to focus solely on the $99.7 \%$ of phase errors which were $2 \pi$ rad below the expected value. Images of M202 melanoma cells contained far fewer phase unwrapping errors after phase unwrapping via the Goldstein method, with only $0.06 \%$ of pixels being phase wrapped, and $>99.9 \%$ being $2 \pi$ rad below the expected value. This makes the phase unwrapping problem for these images a two-class classification problem, which is readily amenable to the use of statistical classifier techniques. ${ }^{36}$

We first segmented these images using LDA based on the manual classification results and 17 pixelwise statistics for
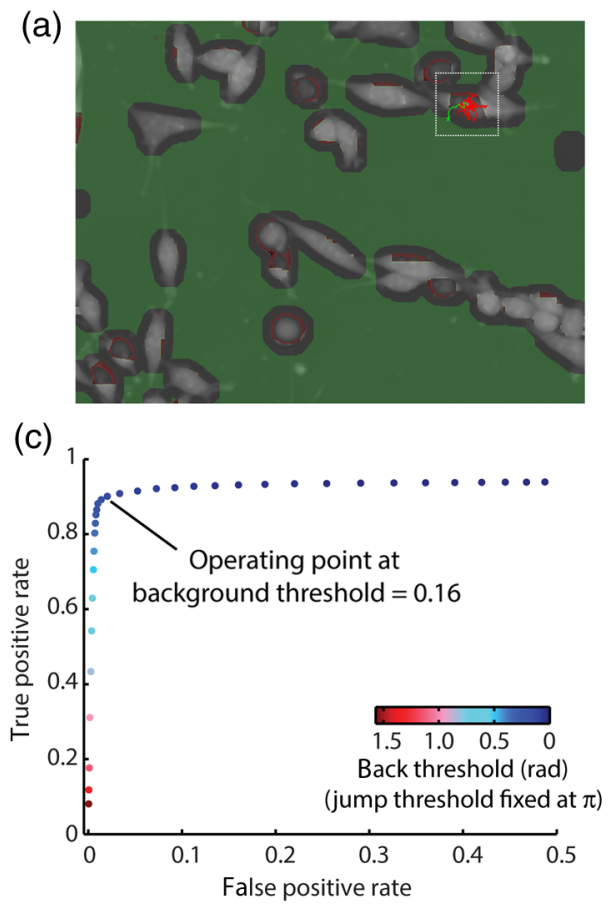

each image, 12 based on the raw phase image itself, 3 based on the phase quality magnitude image, and 2 based on the intensity image [Figs. 1(a)-1(e)]. Most image statistics are based on linear image filters chosen to highlight the edges of cells and phase-wrapped regions in the raw phase image. We used the natural logarithm of the PDV and phase quality magnitude to provide an image that more closely matched the dynamic range of the others in the set.

We performed LDA to classify pixels into two classes: (1) pixels lying on the edge of phase-wrapped regions and (2) correctly unwrapped pixels and pixels in the interior of phase-wrapped regions. This gave a superior performance relative to LDA for separation of phase-wrapped interiors from correctly unwrapped regions, likely due to the presence of strong features at the edges of phase wrapped regions and because the interiors of phase-wrapped regions resemble correctly unwrapped pixels. LDA provides a set of coefficients to the 17 input images that maximizes the ratio of between-class difference to the withinclass difference. ${ }^{29}$ The final LDA image resulting from this analysis is, therefore, a linear combination of all 17 input images. We observed a fair agreement between manually marked edges and those with an LDA-determined probability of being in the edge class, $p_{\text {edge }}$, of $>0.5$ [Fig. 1(f)].

Phase unwrapping, however, requires determination of the interior, wrapped pixels as well as the edges, and most LDAdetermined edges did not define a continuous boundary. To complete the LDA boundaries, we used a marked watershed

(b)

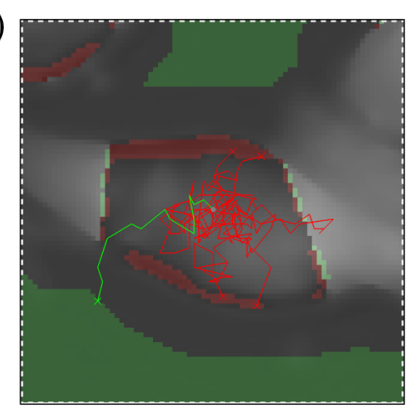

(d)

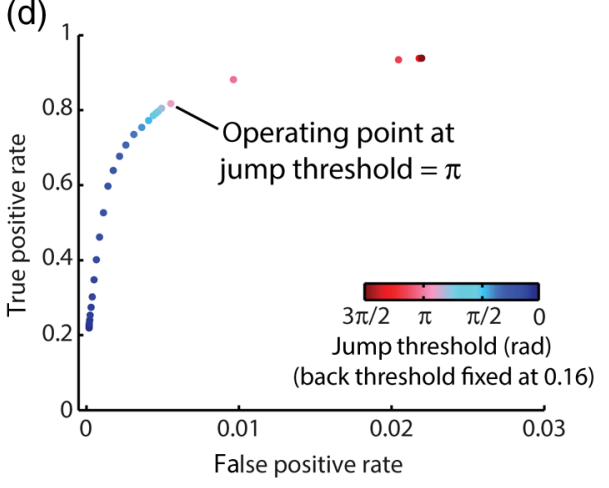

Fig. 2 Random walker (RW)-based image segmentation for phase unwrapping. (a and b) marked phase image of mouse fibroblasts with sample unbiased RW agent paths. Green shows automatically determined known-good regions (background or falling edge), red shows automatically determined knownbad regions (phase level < background threshold, or rising edge). Green line shows a RW path originating from the center, gray marked pixel which terminates at a known-good pixel. Red lines show paths terminating as a known-bad pixel. Based on the five shown paths, the pixel in question would have a probability of $4 / 5$ of belonging to the "bad" (phase-wrapped) group. (c) Algorithm performance as a function of background threshold with jump threshold fixed to $\pi$ rad [based on operating point from panel (d)], used to determine when a pixel is too far below the background level to represent data from a biological sample. (d) Algorithm performance as a function of jump threshold with background threshold fixed to $0.16 \mathrm{rad}$ [based on operating point from panel (c)], used to determine when a given edge is rising or falling. 
algorithm to segment the LDA class probability images. We performed genetic optimization of the input LDA coefficients, which improved the performance moderately and defines the receiver operator characteristic (ROC) with $\mathrm{AUC}=0.923$ [Fig. 1(g)].

\subsection{Random Walker Segmentation}

For most cells imaged in aqueous media $n_{\text {cell }}>n_{\text {media }}$ (for example, Ref. 40) and so the phase shift relative to the background will be greater than zero. This assumption, plus the observation that, in our manually corrected datasets, most residual phase unwrapping errors involve phase being one wavelength ( $2 \pi \mathrm{rad})$ too low, enable us to define boundaries of good and bad regions in the partially wrapped phase data [Figs. 2(a) and 2(b)]. We used a method based on simulating a random walk to use these assumptions to automatically mask phase-wrapped regions.

(a)

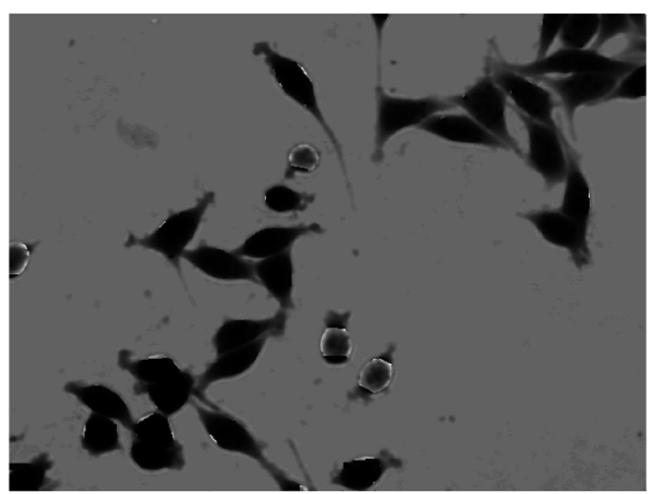

(b)

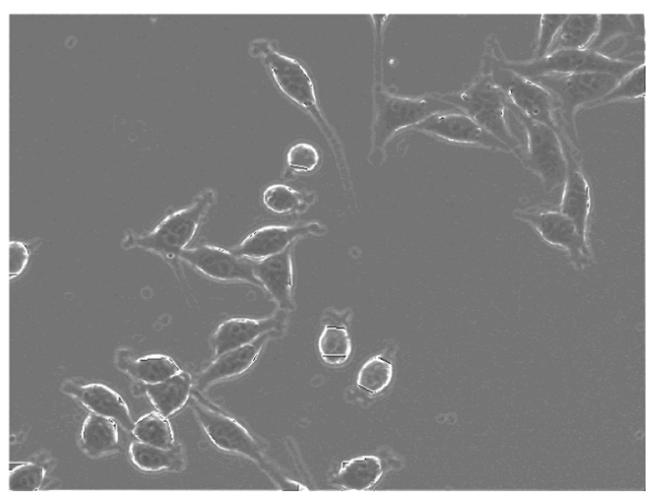

(c)

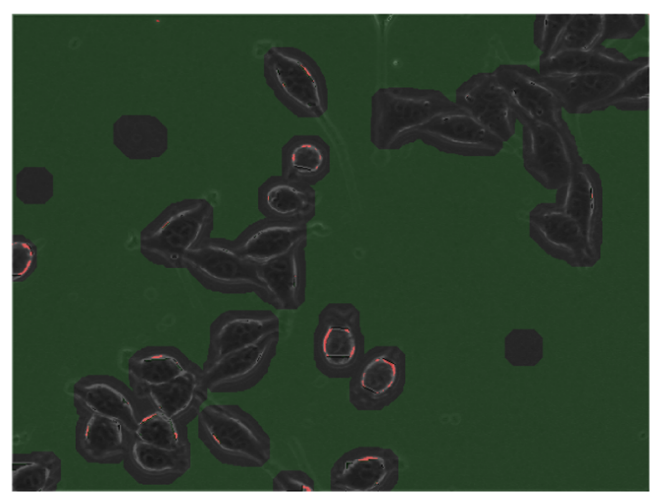

Fig. 3 Combination of LDA and RW methods. (a) Phase image. (b) LDA image. (c) LDA image with superimposed, automatically determined known-good (green) and known-bad (red) regions.
Two methods were used to perform the random walk. In the first, a RW agent method, 20 RW agents were released from each pixel with initially unknown status. Each agent then performed a random walk until it hit a known-good or known-bad pixel [Figs. 2(a) and 2(b)]. The status of the initially unknown pixel was determined by the ratio of "bad" paths to total paths, with a threshold of 0.3 to 0.9 typically used to phaseunwrap images. In the five example paths shown in Figs. 2(a) and 2(b), four out of five RW agent paths terminate at a knownbad pixel, thus the probability that the start pixel is phase wrapped and this pixel would be classified as "bad" is $4 / 5=0.8$. This method, however, is slow, typically requiring 40 to $90 \mathrm{~s}$ /image with $20 \mathrm{RW}$ agents on a single $2.4 \mathrm{GHz}$ Intel Xeon core and potentially inaccurate as it relies on a limited number of random samples.

A faster and more accurate method is to use the random walk image segmentation method presented by Grady. ${ }^{30}$ This method uses the fact that the RW problem is exactly the solution to the Dirichlet problem with boundary conditions at the initially known pixels to solve the RW problem with a single matrix equation solution. This method can also be modified with weights at each pixel based on the intensity of an input image to effectively separate regions with very different intensity values. ${ }^{30}$ Including preclassification of known-good and knownbad regions, this approach requires $\sim 1.5 \mathrm{~s} /$ image on a single 2.4-GHz Intel Xeon core relative to 40 to $90 \mathrm{~s} /$ image with the RW agent approach.

The main controlling parameter for RW image segmentation is the threshold for how far below the background a pixel has to be to be considered a "bad" pixel [Fig. 2(c)]. If this value is set too low, the false positive rate increases rapidly. Best results (minimized misclassification errors) were achieved with a background threshold of $0.16 \mathrm{rad}$. The other parameter is the jump threshold, which determines how much of a jump from one pixel to the next is considered a phase unwrapping artifact [Fig. 2(d)]. This value was set to $\pi$ rad, but moderately larger or smaller values do not have a large effect on the number of false positive errors.

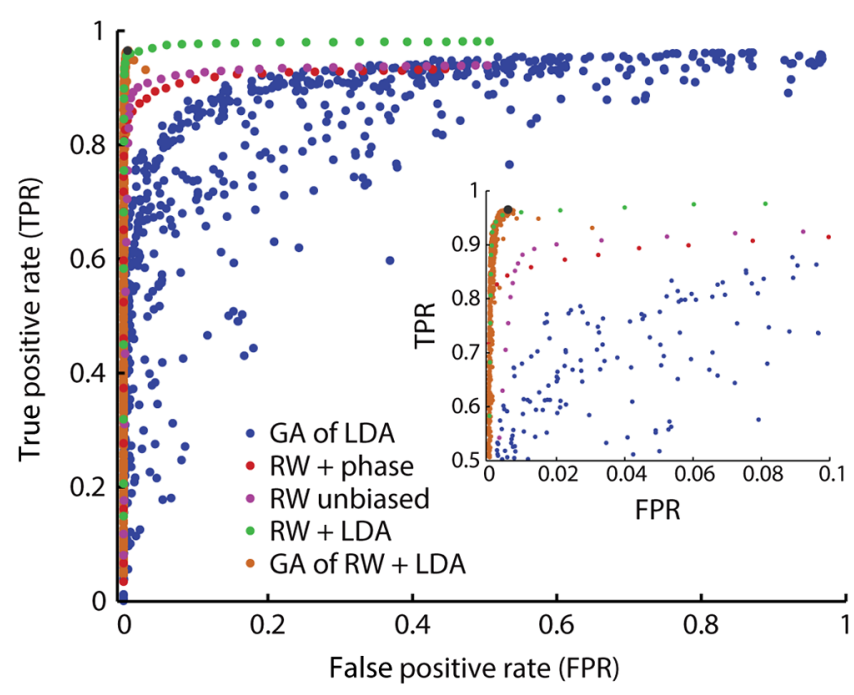

Fig. 4 Receiver-operator characteristics for phase unwrapping methods. The application of a GA to optimize the hybrid RW + LDA method gives the best results, with an AUC of 0.999. AUC GA + LDA $=0.923$, $\mathrm{RW}+$ phase $=0.945$, unbiased $\mathrm{RW}=0.947, \mathrm{RW}+\mathrm{LDA}(\mathrm{HRL})=0.984$, $\mathrm{GA}$ of $\mathrm{RW}+\mathrm{LDA}(\mathrm{HRL})=0.999$. The chosen operating point, which minimizes total error rate, is shown in black. 


\subsection{Linear Discriminant Analysis + Random Walker}

The Grady RW algorithm can be "biased" to follow natural edges in an image, with edge weights based on a Gaussian weighting function. ${ }^{30}$ We tested three inputs to the RW weighting function: (1) no input (unbiased random walk), (2) the raw phase image, and (3) the LDA class probability image. The edges of phase wrapped regions in the LDA image are highlighted much more clearly than in the raw phase image (Fig. 3), indicating that LDA class probabilities may serve as effective weights for image segmentation. We find that the raw phase image actually results in worse segmentation than the unbiased RW algorithm (Fig. 4) with an AUC of 0.945 for the ROC when the background threshold is varied, compared to 0.947 for the unbiased RW. As expected, the use of the LDA class probability image (Fig. 3) to bias the RW algorithm results in a significant improvement, with an AUC of 0.984.

The final refinement performed was to run a genetic optimization on the LDA coefficients and background threshold for the

(a)

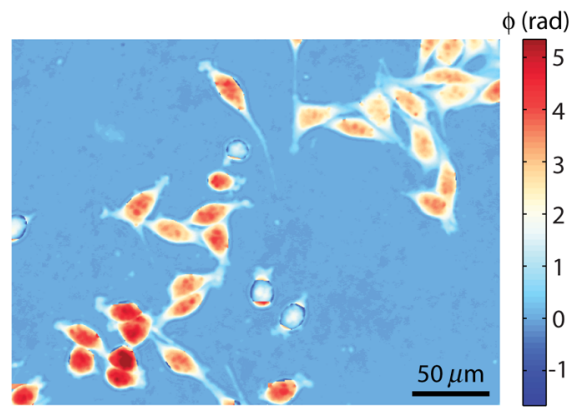

(b)

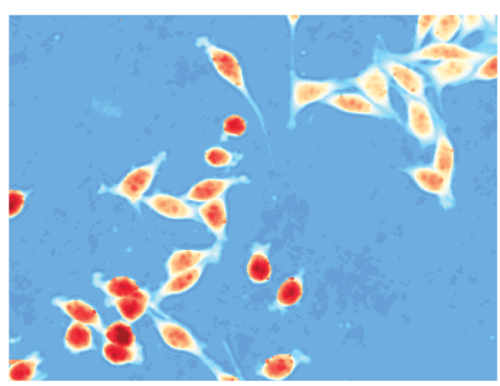

(c)

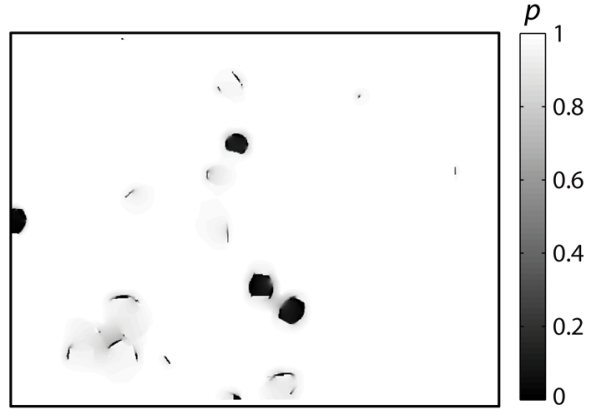

HRL method. This resulted in the best overall AUC (0.999) for the training data set, achieving a very high true positive rate with a very low false positive rate. The optimal operating point based on these results, which minimizes the total misclassification error, achieves a true positive rate of 0.965 , a false positive rate of 0.006 , and an overall misclassification rate of $0.6 \%$ on the training dataset.

\subsection{Final Results}

We applied the optimized HRL method to a set of 50 manually unwrapped test images separate from those used for classifier training (Fig. 5). The results show good overall correction of phase errors [Figs. 5(a) and 5(b)]. The RW algorithm returns the probability that a given pixel is phase wrapped [or, as in Fig. 5(c) the complement of this, the probability that a pixel is not phase wrapped]. This conforms well with the expected manual results with a threshold probability of 0.5 . Results for all images in the manually corrected training and test datasets

(d)

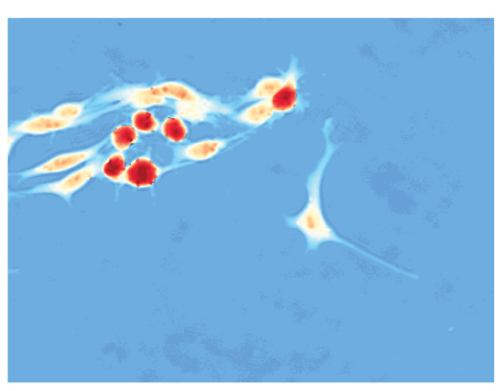

(e)
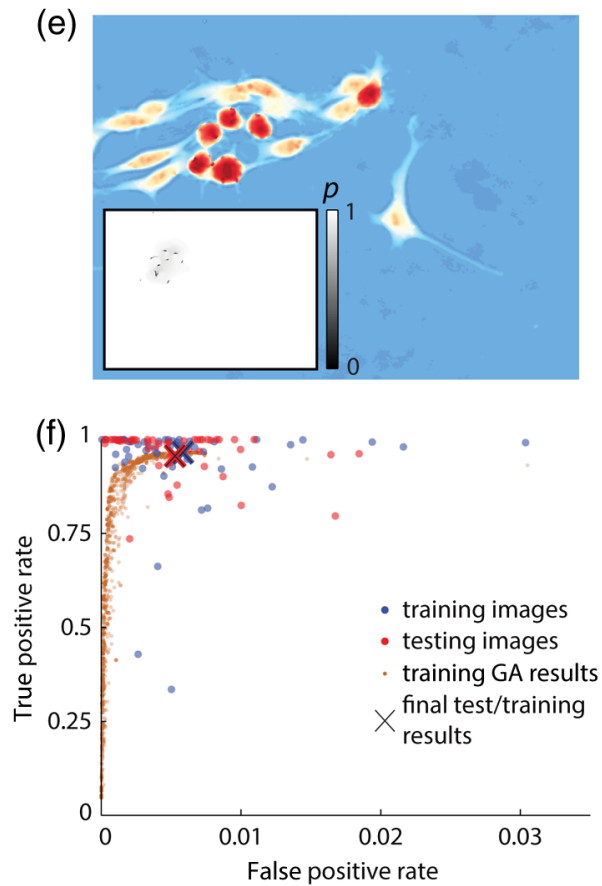

Fig. 5 Final results of hybrid random walk-linear (HRL) algorithm. (a) Sample original phase image and (b) final HRL output image. (c) Pixelwise probability, $p$, of an LDA-biased RW agent reaching a knowngood pixel (i.e., probability that a given pixel is not phase-wrapped). ( $d$ and $e$ ) sample original and final HRL output images showing low false positive rate when there are few errors in the input image. Inset in (e) shows the same pixelwise probability, $p$, presented in panel (c). (f) Error rates for all 50 individual images in the manually phase-unwrapped training dataset used to train the classifier and for 50 individual images used to test the classifier performance. GA results for aggregate of all training data set images are shown for reference. Final overall results for both test and training datasets are each marked with an $x$. Scale in (b), (d), and (e) is the same as shown in (a). 

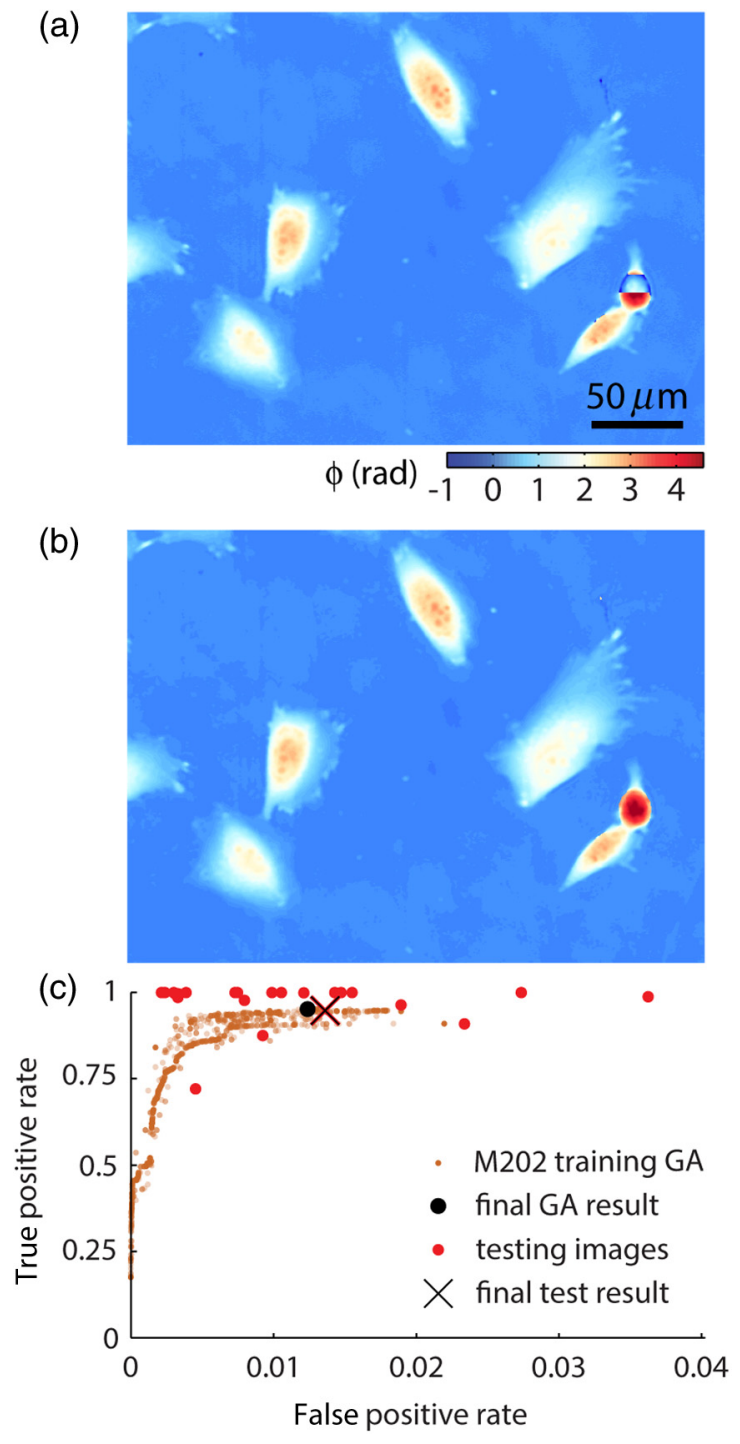

Fig. 6 Results of HRL algorithm applied to M202 human melanoma cell training and test data sets. (a) Sample original phase image of M202 melanoma cells. (b) Final HRL output image. Scale in (b) is the same as shown in (a). (c) GA results for aggregate of all training data set images (orange) with error rates for all 50 individual images used to test the classifier performance. GA results for M202 melanoma cells show an AUC of 0.998. Final overall results for both GA optimization on the training dataset and the HRL method applied to the test dataset are each marked.

show good overall performance with the optimally chosen parameters from the GA, including background threshold = $0.16 \mathrm{rad}$. The test data results compare well with the results on the training dataset, with a true positive rate equal to 0.956 , a false positive rate equal to 0.005 , and an overall misclassification rate of $0.6 \%$.

Finally, we applied the HRL method to a separate data set consisting of QPI data of M202 human melanoma cells. As shown in Fig. 6, performance with this cell type was comparable to performance on the L-cell fibroblast dataset, with AUC = 0.998 after genetic optimization on a training data set of 50 images. Performance on the test dataset of an additional 50 images was also high, with a true positive rate of 0.947 , a false positive rate of 0.014 , and an overall misclassification rate of $1.4 \%$.

\section{Discussion}

We have demonstrated that the HRL method after genetic optimization has the best phase unwrapping performance with a high final AUC of 0.999 (Fig. 4). The overall low error rate was reproduced well on a second test data set (Fig. 5) and on a second cell type (Fig. 6), showing that the HRL algorithm is a consistent, reliable method for phase unwrapping of biological QPI data. This method is primarily useful for biological samples on flat substrates, but the general approach would be applicable for any phase sample where the standard algorithms have failed and pixels can be automatically labeled based on a set of image features. Future work will examine how the HRL algorithm could be made more efficient by utilizing other classifiers and/or reducing the statistic set used for the LDA classifier to further reduce the computational time.

The general procedure of the HRL method can be adapted to phase unwrapping of other datasets. The general method is to (1) choose image statistics to use in the LDA, giving preference to those that will emphasize the edges of phase-wrapped regions (e.g., phase quality, ${ }^{41}$ fringe modulation, ${ }^{31}$ or edge detection filters $\left.^{34}\right)$, (2) perform manual phase unwrapping for use as a training dataset, (3) perform LDA to separate edges of marked regions from all other pixels, (4) use image features as premarked inputs to RW segmentation, ${ }^{30}$ biased by the weights output by LDA, and (5) as an optional last step, tune LDA coefficients, for example, using a genetic search algorithm. We also note that different components of the HRL algorithm described here can be repurposed for other data processing tasks. For example, the combination of LDA plus a biased random walk would be an effective general method to segment images automatically, for example, to segment cells from background as an alternative to the widely used watershed algorithm. ${ }^{34}$ In this case, the LDA probability image would be used to combine multiple image features to enhance the precision of finding object or cell edges, eliminating the necessity for timeconsuming manual image segmentation. As in the present study, a GA could then be used to further refine the classifier performance for the system of interest. Therefore, we expect that the HRL approach presented here is generally applicable to a variety of image classification problems, beyond phase unwrapping of biological QPI data.

\section{Acknowledgments}

This study is supported by National Institutes of Health awards K25CA157940 (TAZ), R01CA90571, R01CA156674, R01GM073981, R01GM114188 (MAT), R01CA185189 (MAT and JR), and R01GM094388 (JR), by a NantWorks, LLC-University of California Discovery Biotechnology Award (Bio0710663) (MAT), by a California Institute for Regenerative Medicine Basic Biology 1 and Tools and Technologies III Awards (RB1-01397 and RT3-07678) (MAT), by the Broad Stem Cell Research Center at UCLA (TAZ and MAT), and by a Translational Acceleration Grant from the Caltech-UCLA Joint Center for Translational Medicine (www.jctm.caltech.edu) (MAT).

\section{References}

1. T. A. Zangle and M. A. Teitell, "Live-cell mass profiling: an emerging approach in quantitative biophysics," Nat. Methods 11(12), 1221-1228 (2014).

2. G. Popescu, Quantitative Phase Imaging of Cells and Tissues, McGrawHill, New York, New York (2011). 
3. B. Rappaz et al., "Noninvasive characterization of the fission yeast cell cycle by monitoring dry mass with digital holographic microscopy," $J$. Biomed. Opt. 14(3), 034049 (2009).

4. B. Kemper et al., "Investigation of living pancreas tumor cells by digital holographic microscopy," J. Biomed. Opt. 11(3), 034005 (2006).

5. B. Kemper et al., "Simplified approach for quantitative digital holographic phase contrast imaging of living cells," J. Biomed. Opt. 16(2), 026014 (2011).

6. J. Chun et al., "Rapidly quantifying drug sensitivity of dispersed and clumped breast cancer cells by mass profiling," Analyst 137(23), 5495-5498 (2012).

7. J. Reed et al., "Rapid, massively parallel single-cell drug response measurements via live cell interferometry," Biophys. J. 101(5), 1025-1031 (2011).

8. M. Mir et al., "Highly sensitive quantitative imaging for monitoring single cancer cell growth kinetics and drug response," PLoS One 9(2), e89000 (2014).

9. M. Mir et al., "Optical measurement of cycle-dependent cell growth," Proc. Natl. Acad. Sci. U. S. A. 108(32), 13124-13129 (2011).

10. T. A. Zangle et al., "Quantifying biomass changes of single CD8+ T cells during antigen specific cytotoxicity," PLoS One 8(7), e68916 (2013).

11. P. Bon et al., "Optical detection and measurement of living cell morphometric features with single-shot quantitative phase microscopy," $J$. Biomed. Opt. 17(7), 076004 (2012).

12. Y. Park et al., "Measurement of red blood cell mechanics during morphological changes," Proc. Natl. Acad. Sci. U. S. A. 107(15), 67316736 (2010).

13. Y. Park et al., "Refractive index maps and membrane dynamics of human red blood cells parasitized by Plasmodium falciparum," Proc. Natl. Acad. Sci. U. S. A. 105(37), 13730-13735 (2008).

14. N. T. Shaked et al., "Quantitative microscopy and nanoscopy of sickle red blood cells performed by wide field digital interferometry," $J$. Biomed. Opt. 16(3), 030506 (2011).

15. D. C. Ghiglia and M. D. Pritt, Two-Dimensional Phase Unwrapping: Theory, Algorithms, and Software, John Wiley \& Sons, New York, New York (1998).

16. A. Baldi, "Two-dimensional phase unwrapping by quad-tree decomposition," Appl. Opt. 40(8), 1187-1194 (2001).

17. R. M. Goldstein, H. A. Zebker, and C. L. Werner, "Satellite radar interferometry: two-dimensional phase unwrapping," Radio Sci. 23(4), 713720 (1988).

18. M. A. Herráez, M. J. L. Burton, and M. A. Gdeisat, "Fast two-dimensional phase-unwrapping algorithm based on sorting by reliability following a noncontinuous path," Appl. Opt. 41(35), 7437-7444 (2002).

19. T. J. Flynn, "Two-dimensional phase unwrapping with minimum weighted discontinuity," J. Opt. Soc. Am. A 14(10), 2692-2701 (1997).

20. D. C. Ghiglia and L. A. Romero, "Robust two-dimensional weighted and unweighted phase unwrapping that uses fast transforms and iterative methods," J. Opt. Soc. Am. A 11(1), 107-117 (1994).

21. M. A. Navarro et al., "Fast two-dimensional simultaneous phase unwrapping and low-pass filtering," Opt. Express 20(3), 2556-2561 (2012).

22. R. A. Hoebe et al., "Controlled light-microscopy reduces photobleaching and phototoxicity in fluorescence live-cell imaging," Nat. Biotechnol. 25(2), 249-253 (2007).

23. A. Khodjakov and C. L. Rieder, "Imaging the division process in living tissue culture cells," Methods 38(1), 2-16 (2006).

24. S. A. Telenkov and A. Mandelis, "Fourier-domain biophotoacoustic subsurface depth selective amplitude and phase imaging of turbid phantoms and biological tissue," J. Biomed. Opt. 11(4), 044006 (2006).

25. E. Watanabe, T. Hoshiba, and B. Javidi, "High-precision microscopic phase imaging without phase unwrapping for cancer cell identification," Opt. Lett. 38(8), 1319-1321 (2013).

26. T. E. Gureyev and K. A. Nugent, "Rapid quantitative phase imaing using the transport of intensity equation," Opt. Commun. 133, 339346 (1997).

27. C. Zuo et al., "Noninterferometric single-shot quantitative phase microscopy," Opt. Lett. 38(18), 3538 (2013).
28. H. Pham et al., "Off-axis quantitative phase imaging processing using CUDA: toward real-time applications," Biomed. Opt. Express 2(7), 1781-1793 (2011).

29. A. H. Fielding, Cluster and Classification Techniques for the Biosciences, Cambridge University Press, Cambridge (2007).

30. L. Grady, "Random walks for image segmentation," IEEE Trans. Pattern Anal. Mach. Intell. 28(11), 1768-1783 (2006).

31. C. Wells and J. C. Wyant, "Fringe modulation characterization for a phase shifting imaging ellipsometer," Proc. SPIE 3134, 466-474 (1997).

32. J. N. Sondergaard et al., "Differential sensitivity of melanoma cell lines with BRAFV600E mutation to the specific Raf inhibitor PLX4032," J. Transl. Med. 8, 39 (2010).

33. T. A. Zangle, M. A. Teitell, and J. Reed, "Live cell interferometry quantifies dynamics of biomass partitioning during cytokinesis," PLoS One 9(12), e115726 (2014).

34. R. C. Gonzales, R. E. Woods, and S. L. Eddins, Digital Image Processing Using Matlab, 2nd ed., Gatesmark Publishing, Knoxville, Tennessee (2009).

35. B. Spottiswoode, " 2 D Phase Unwrapping Algorithms," Matlab Central File Exchange, 22504, http://www.mathworks.com/matlabcentral/ fileexchange/22504-2d-phase-unwrapping-algorithms (23 February 2008).

36. A. R. Webb, Statistical Pattern Recognition, John Wiley \& Sons, Ltd., Hoboken, New Jersey (2002).

37. W. Dwinnell, "LDA: Linear Discriminant Analysis," Matlab Central File Exchange, 29673, http://www.mathworks.com/matlabcentral/ fileexchange/29673-lda--linear-discriminant-analysis (11 December 2010).

38. P. G. Doyle and L. L. Snell, Random Walks and Electric Networks, Mathematical Association of America, Washington, DC (1984).

39. S. Kakutani, "Markov processes and the Dirichlet problem," Proc. Japan Acad. 21, 227-233 (1945).

40. R. Barer and S. Joseph, "Refractometry of living cells. Part I. Basic principles," Q. J. Microsc. Sci. 95, 399-423 (1954).

41. Y. Lu, W. Zhao, and X. Zhang, "Quality map generation in two-dimensional phase unwrapping process by using edge detection techniques," in Vision Sensors and Edge Detection, G.-F. Francisco, Ed., Intech, Rijeka, Croatia (2010).

Diane N. H. Kim is a graduate student in the Bioengineering Department at the University of California, Los Angeles. She received her BS in biomedical engineering from Columbia University. Her research interests are immunology and quantitative phase imaging.

Michael A. Teitell holds the Latta Endowed Chair in Pathology and is a professor of pathology and laboratory medicine, pediatrics, and bioengineering at UCLA. He is a director of the Cancer Nanotechnology Program in the Jonsson Cancer Center and was elected to the American Society of Clinical Investigators. His research lab collaboratively developed a photothermal nanoblade for large cargo transfers into cells and a live cell interferometer to dissect heterogeneous tumor cell responses to drugs.

Jason Reed is an assistant professor in the Department of Physics at Virginia Commonwealth University and a member of the Developmental Therapeutics Program at the VCU Massey Cancer Center. His research group develops novel approaches for biomaterials characterization and for real-time analysis of living systems, with applications in drug development and molecular diagnostics. He received his $\mathrm{PhD}$ from New York University and his AB from Harvard College.

Thomas A. Zangle is an assistant professor in the Department of Bioengineering at UCLA. His work focuses on the application of quantitative phase imaging to measure the biophysical properties of biological samples with applications in cancer and stem cell biology. $\mathrm{He}$ received his $\mathrm{PhD}$ in mechanical engineering from Stanford University for work focusing on experimental, theoretical, and computational descriptions of transport in microfluidic-nanofluidic systems and his AB from Dartmouth College. 